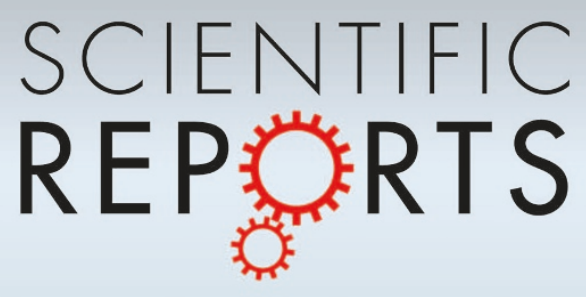

OPEN

SUBJECT AREAS:

COMPUTATIONAL

SCIENCE

FLUID DYNAMICS

COMPUTATIONAL BIOPHYSICS

INTERSTELLAR MEDIUM

Received

31 January 2013

Accepted

15 October 2013

Published

31 October 2013

Correspondence and requests for materials should be addressed to

M.M. (mmendoza@ ethz.ch)

\section{Flow Through Randomly Curved Manifolds}

\author{
M. Mendoza' ${ }^{1}$ S. Succi ${ }^{2}$ \& H. J. Herrmann ${ }^{3,4}$
}

'ETH Zürich, Computational Physics for Engineering Materials, Institute for Building Materials, Wolfgang-Pauli-Strasse 27, HIT, CH8093 Zürich (Switzerland), ${ }^{2}$ Istituto per le Applicazioni del Calcolo C.N.R., Via dei Taurini, 1900185 , Rome (Italy), and Freiburg Institute for Advanced Studies, Albertstrasse, 19, D-79104, Freiburg, (Germany), ${ }^{3}$ ETH Zürich, Computational Physics for Engineering Materials, Institute for Building Materials, Schafmattstrasse 6, HIF, CH-8093 Zürich (Switzerland), ${ }^{4}$ Departamento de Física, Universidade Federal do Ceará, Campus do Pici, 60455-760 Fortaleza, Ceará, (Brazil).

We present a computational study of the transport properties of campylotic (intrinsically curved) media. It is found that the relation between the flow through a campylotic media, consisting of randomly located curvature perturbations, and the average Ricci scalar of the system, exhibits two distinct functional expressions, depending on whether the typical spatial extent of the curvature perturbation lies above or below the critical value maximizing the overall scalar of curvature. Furthermore, the flow through such systems as a function of the number of curvature perturbations is found to present a sublinear behavior for large concentrations, due to the interference between curvature perturbations leading to an overall less curved space. We have also characterized the flux through such media as a function of the local Reynolds number and the scale of interaction between impurities. For the purpose of this study, we have also developed and validated a new lattice Boltzmann model.

M any systems in Nature present either spatial curvature (e.g. curved space due to presence of stars ${ }^{1}$ or flow on soap films $\mathrm{s}^{2}$ ) or geometric confinement constraining the degrees of freedom of particles moving on such media, e.g. solar photosphere ${ }^{3}$, flow between two rotating cylinders and spheres ${ }^{4-6}$, hemodynamics through deformable vessels ${ }^{7}$, fusion plasmas ${ }^{8}$, and flow through porous media ${ }^{9}$, to name but a few. In general, these systems force a fluid to move along non-straight trajectories (curved geodesics), leading to the upsurge of non-inertial forces. We will denote such systems as Campylotic, from the greek word $\kappa \alpha \mu \tau \hat{i} \lambda o \varsigma$ for curved, media. Due to the arbitrary trajectories that particles through a campylotic medium can take, depending on the complexity of the curved space, the flow through these media can present very unusual new transport properties. Campylotic media play a prominent role in all applications where metric curvature has a major impact on the flow structure and topology; biology, astrophysics and cosmology offering perhaps the most natural examples. Indeed, the flow through some specific campylotic media has already been studied, e.g. Taylor-Couette flow and flow through porous media. The Taylor-Couette flow was originally formulated for the case of two concentric, rotating cylinders ${ }^{4,5}$, and later extended to the case of spheres $^{6,10}$. On the other hand, continuum descriptions of flow through homogeneous porous media have been already treated as changes in the fluid trajectories, by resorting to the concept of tortuosity, which is the ratio between the straight line distance to the curved path length (due to the presence of obstacles) between two points in the medium. The tortuosity is directly related to the permeability and porosity of the medium ${ }^{9}$. However, the flow through other complicated structures, like randomly located stars (which is an intrinsically curved space) or many biological systems, to the best of our knowledge, has never systematically been addressed before on quantitative grounds.

Since, in general, this class of flows lacks analytical solutions, their study is inherently dependent on the availability of appropriate numerical methods. Flows in complex geometries, such as cars or airplanes, make a time-honored mainstream of computational fluid dynamics (CFD), a discipline which has made tremendous progress for the last decades ${ }^{11,12}$. However, campylotic media set a major challenge even to the most sophisticated CFD methods, because the geometrical complexity is often such to command very high spatial accuracy to resolve the most acute metric and topological features of the flow. Therefore, in this work, we also present a new lattice kinetic scheme that can handle flows in virtually arbitrary complex manifolds in a very natural and elegant way, by resorting to a covariant formulation of the lattice Boltzmann (LB) kinetic equation in general coordinates and for curved spaces.

As an additional feature, complex boundary conditions related with a specific geometry, e.g. surface of a sphere, or more sophisticated ones, like Möbius bands and the Klein bottle, can be treated exactly by cubic cells in the 
contravariant coordinate frame, thereby avoiding the staircase approximations which would result from the use of cubic cells associated with euclidean geometry. For instance, in spherical coordinates, we can construct a lattice with cubic cells of volume $\delta r \times \delta \theta \times \delta \phi$, being $(r, \theta, \phi)$ the coordinates of each grid point. This feature can also be used to model the microscopic geometry of a porous medium by computing, analytically or numerically, the coordinate system that parametrizes the pore structure, and thus, to avoid the staircase approximation of the obstacles. The method is validated quantitatively for very simple campylotic media with geometrical confinements by calculating the critical Reynolds number for the onset of the Taylor-Couette instability in concentric cylinders and spheres ${ }^{5,6,10,13}$, and applied to the case of two concentric tori.

In this work, by using the new numerical scheme, we simulate the flow through campylotic media consisting of randomly distributed spatial curvature perturbations (see Fig. 1), in the laminar regime. The flow is characterized by the number of curvature perturbations and the average Ricci scalar of the space. The campylotic media explored in this work are static, in the sense that the metric tensor and curvature are prescribed at the outset once and for all, and do not evolve self-consistently with the flow. The latter case, which is a major mainstream of current numerical relativity ${ }^{14-17}$, especially in the turbulent regime, makes a very interesting subject for future extensions of this work.

\section{Results}

Kinetic theory in general manifolds. In order to study the campylotic media, we develop a lattice Boltzmann approach for curved spaces in general coordinates, taking into account the metric tensor $g_{i j}$ and the Christoffel symbols $\Gamma_{k j}^{i}$. The former characterizes the way to measure distances in space, while the latter is responsible for the non-inertial forces. The corresponding hydrodynamic equations can be obtained by replacing the partial derivatives by covariant ones, in both, the mass continuity and the momentum conservation equations. After some algebraic manipulations, the hydrodynamic equations read as follows: $\partial_{t} \rho+\left(\rho u^{i}\right)_{; i}=0$, and $\partial_{t}\left(\rho u^{i}\right)+T_{; j}^{i j}=0$, where the notation ${ }_{; i}$ denotes the covariant derivative with respect to spatial component $i$ (see SI). The energy tensor $T^{i j}$ is given by, $T^{i j}=P g^{i j}+\rho u^{i} u^{j}-\mu\left(g^{l j} u_{; l}^{i}+g^{i l} u_{; l}^{j}+g^{i j} u_{; l}^{l}\right)$, where $P$ is the hydrostatic pressure, $u^{i}$ the $i$-th contravariant component of the velocity, $g^{i j}$ the inverse of the metric tensor, $\rho$ is the density of the fluid, and $\mu$ is the dynamic shear viscosity.

Since lattice Boltzmann methods are based on kinetic theory, we construct our model by writing the Maxwell-Boltzmann distribution and the Boltzmann equation for general manifolds. The former takes the form ${ }^{18}$ :

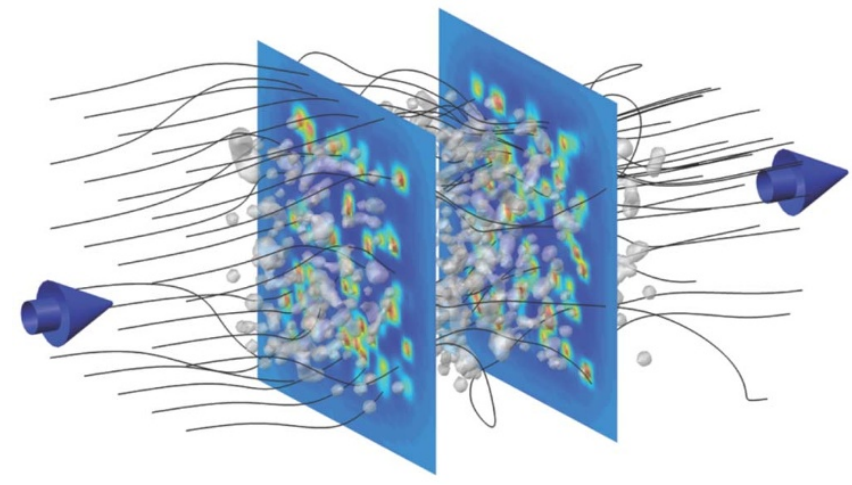

Figure 1 Streamlines of a three-dimensional fluid moving through a campylotic medium. The colors denote the scalar of curvature $R^{\prime}$ (blue and red for low and high values, respectively). The gray bubbles isosurfaces stand at $1 / 5$ of the maximum curvature of the system.

$$
f^{\mathrm{eq}}=\frac{\sqrt{g} \rho}{(2 \pi \theta)^{3 / 2}} \exp \left[-\frac{1}{2 \theta} g_{i j}\left(\xi^{i}-u^{i}\right)\left(\xi^{j}-u^{j}\right)\right],
$$

where $g$ is the determinant of the metric $g_{i j}$, and $\theta$ is the normalized temperature. The macroscopic and microscopic velocities, $u^{i}$ and $\xi^{i}$ are both normalized with the speed of sound $c_{s}=\sqrt{k_{B} T_{0} / m}, k_{B}$ being the Boltzmann constant, $T_{0}$ the typical temperature, and $m$ the mass of the particles. Note that the metric tensor appears explicitly in the distribution function, due to the fact that the kinetic energy is a quadratic function of the velocity, $u^{i} u_{i}=g_{i j} u^{i} u^{j}$. To recover the macroscopic fluid dynamic equations, we have to extract the moments from the equilibrium distribution function. The four first moments of the Maxwellian distribution function on a manifold are given by,

$$
\begin{gathered}
\rho=\int f d \xi \quad, \quad \rho u^{i}=\int f \xi^{i} d \xi, \\
\rho \theta g^{i j}+\rho u^{i} u^{j}=\int f \xi^{i} \xi^{j} d \xi \quad, \\
\rho \theta\left(u^{i} g^{j k}+u^{j} g^{i k}+u^{k} g^{i j}\right)+\rho u^{i} u^{j} u^{k}=\int f \xi^{i} \xi^{j} \xi^{k} d \xi .
\end{gathered}
$$

These moments are sufficient to reproduce the mass and the momentum conservation equations. Here, for simplicity we have used $d \xi$ to denote $d \xi^{1} d \xi^{2} d \xi^{3}$ and the Jacobian of the integration is already included in the Maxwell Boltzmann distribution, through the determinant term $\sqrt{g}$.

In the absence of external forces, in the standard theory of the Boltzmann equation, the single particle distribution function $f\left(x^{i}, \xi^{i}, t\right)$ evolves, according to the equation, $\partial_{t} f+\xi^{i} \partial_{i} f=\mathcal{C}(f)$, where $\mathcal{C}$ is the collision term, which, using the BGK approximation, can be written as, $\mathcal{C}=-(1 / \tau)\left(f-f^{\text {eq }}\right)$, with the single relaxation time $\tau$. This equation can be obtained from a more general expression, $d f / d t=\mathcal{C}(f)$, where the total time derivative now includes a streaming term in velocity space due to external forces, $\frac{d f}{d t}=\partial_{t} f+\frac{d x^{i}}{d t} \partial_{i} f+\frac{d p^{i}}{d t} \partial_{p^{i}} f$, with $p^{i}$ the $i$-th contravariant component of the momentum of the particles. Using the definition of velocity, $\xi^{i}=d x^{i} / d t$, and due to the fact that the particles in our fluid move along geodesics, which implies the equation of motion $d p^{i} / d t=-\Gamma_{k l}^{i} p^{k} p^{l}$, we can write the Boltzmann equation, using a procedure based on Ref. 19, as

$$
\partial_{t} f+\xi^{i} \partial_{i} f-\Gamma_{j k}^{i} \xi^{j} \xi^{k} \partial_{\xi^{i}} f=\mathcal{C}(f),
$$

where we have used the definition of the momentum, $p^{i}=m \xi^{i}$. Note that the third term of the left hand side carries all the information on non-inertial forces. The Christoffel symbols and metric tensor are arbitrary and therefore we can model the fluid flow in curved spaces in general coordinates, whose metric tensor is very complicated and/ or only known numerically.

Since the contravariant components of the velocity are free of space-dependent metric factors, they lend themselves to standard lattice Boltzmann discretization of velocity space. All the metric and non-inertial information is conveyed into the generalized local equilibria and forcing term, respectively. These features are key to the LB formulation in general manifolds. As an additional feature, even in flat spaces, complex boundary conditions related to a specific geometry, e.g. surface of sphere, in many cases, can be treated exactly by cubic cells in the contravariant coordinate frame, thereby avoiding stair-case approximations typical of cartesian grids.

Studying the campylotic media. To study a genuinely campylotic medium, consisting of randomly located perturbations of spatial 
intrinsic curvature, we define a coordinate system $(x, y, z)$, such that its metric tensor deviates from the one of a flat space $\left(\delta_{i j}\right)$ in the form: $g_{i j}=\delta_{i j}\left(1-a_{0} \sum_{n=1}^{N} \exp \left(-r_{n} / r_{0}\right)\right)$, where $n$ labels each local curvature perturbation located at $\vec{r}_{n}=\left(x_{n}, y_{n}, z_{n}\right), N$ is the total number of perturbations, $r_{n}=\left|\vec{r}_{n}\right|$, and $r_{0}$ characterizes the size of the deformation. The choice of this metric tensor is to induce an intrinsic curvature in the space at each point $\vec{r}_{n}$. Note that the coefficient $a_{0}$ can be either signed, depending on whether a positive or negative curvature is imposed, respectively. In our study, we have chosen to work with positive values of $a_{0}$, but its sign can be different according to the respective application. In order to understand the effects of the curvature, we will work in the laminar regime to avoid pressure losses due to turbulence. The effects of pressure drops in campylotic media due to fluid turbulence shall be addressed in future work.

The Christoffel symbols are calculated numerically. The flux is calculated by the geometrical relation, $\Phi=\int s \rho u^{x} \sqrt{g^{x x} g} d S$, where $S$ is the cross section at the location where the measurements are taken. Since the fluid dynamic equations only contain the metric tensor and its first derivatives (via the Christoffel symbol), it is natural to expect that the flow could be characterized by a quantity that contains the metric tensor and its first derivatives. Although the Christoffel symbols $\Gamma_{j k}^{i}$ meet this requirement, they are not components of a tensor, and therefore they are not invariant under a coordinate system transformation (physics should not depend on the choice of the coordinate system). An invariant, or tensor, that can be used to characterize the system is the Ricci tensor $R_{i k}$. In this work, we use the Ricci scalar (curvature scalar) which can be calculated from the Ricci tensor, $R_{i j}$, by contraction of the indices, $R^{\prime}$ $=g^{i j} R_{i j}$. The relation between the metric tensor and Christoffel symbols and the Ricci tensor can be found in the SI. For sake of generality and to study our system using dimensionless units, we define a characteristic length $\lambda=(V / N)^{1 / 3}$, where $V=L_{x} L_{y} L_{z}$ is the total volume of our system, and the local Reynolds number $R e=\Phi r_{0} /(\mu S)$. Therefore, the dimensionless size of deformation is defined by $\epsilon=r_{0} / \lambda$. We use a lattice size $L_{x} \times L_{y} \times L_{z}$ of $128 \times 64 \times 64$, and $\tau=1$. All quantities will be expressed in numerical units. To drive the fluid through the medium, we add an external force along the $x$ component, which in all simulations takes the value, $f_{\text {ext }}=5 \times$ $10^{-5}$. The flux in flat space, i.e. in the absence of curvature perturbations is denoted by $\Phi_{0}$.

Shown in Fig. 1, are the velocity streamlines, the Ricci scalar $R^{\prime}$ and the high-curvature locations, represented by gray isosurfaces. Note that the streamlines are very complex, as the flow can orbit around the spheres before continuing its trajectory ${ }^{20,21}$. This effect is due to the complex curvature of the space and has no relation to turbulent vortices, given the low Reynolds number $(R e \sim 1)$. Also we can see how the curvature perturbations interact, creating non-spherical shaped isosurfaces.

Fig. 2 shows the flux deficit $\Phi_{0}-\Phi$, as function of the number of curvature perturbations, $N$. We observe that the flux $\Phi$ decreases with $N$. This effect is due to the interplay between the longer trajectories that particles must take and the acceleration due to the noninertial forces. Note that, in general, for systems with different configurations (e.g. negative $a_{0}$ ), we could expect that the combination of the two effects might lead to higher flux by increasing $N$. We also see that the flux depends linearly on $N$ for low concentration of curvature perturbations, and only sublinearly at higher concentrations. This is due to the fact that at low concentration, the average distance between curvature perturbations is large, and consequently each perturbation adds up as a single modification to the total spatial curvature. However, as the concentration is increased, the curvature perturbations start to interfere with each other and consequently the space becomes less curved (decrease of the overall Ricci curvature).

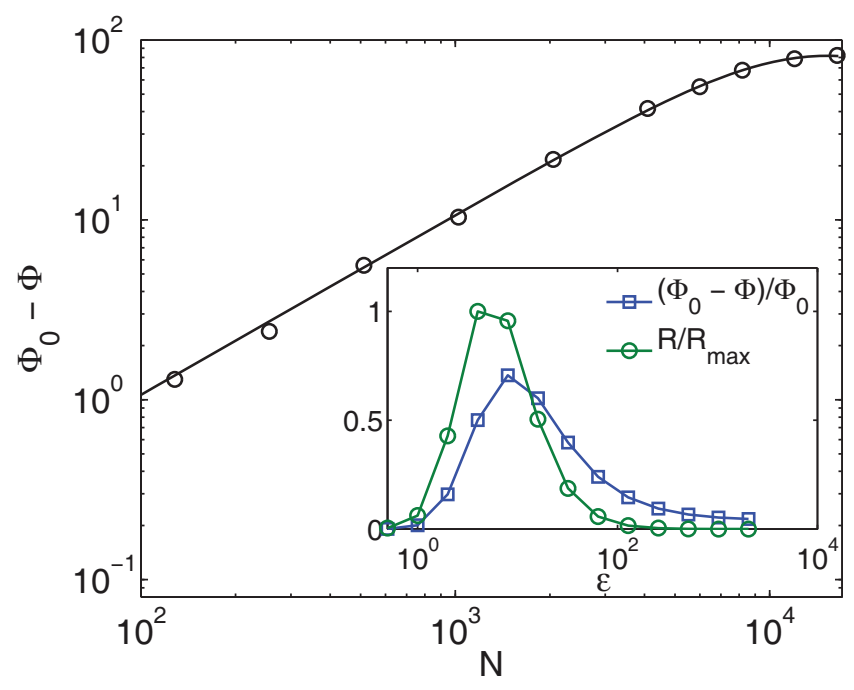

Figure 2 Flux through a campylotic medium consisting of randomly located curvature perturbations. Flux deficit $\Phi_{0}-\Phi$ with respect to the flat case, as a function of the number of curvature perturbations for $a_{0}=$ $0.01, r_{0}=2.0$, and the Re varying between 0.1 and 0.4 . The solid line is the analytical curve according to Eq. (6). Shown in the inset is the normalized average curvature scalar of the space, $R / R_{\max }$, and the normalized reduced flux $1-\Phi_{0} / \Phi$ as a function of $\epsilon(R e \sim 1 \ldots 1000)$. Both Ricci scalar and flux deficit exhibit a maximum at intermediate values of $\epsilon$. Since the two maxima are slightly shifted with respect to each other, the reduced flow as a function of $R$ exhibits two distinct functional expressions (see next Fig. 3).

The flux is found to obey the following law,

$$
\Phi_{0}-\Phi=A_{1} \frac{N / N_{0}}{1+\left(N / N_{0}\right)^{2}},
$$

where $A_{1}=163 \pm 2$ and $N_{0}=(1.54 \pm 0.03) \times 10^{4}$ are fitting parameters. The parameter $N_{0}$ denotes a characteristic number of curvature perturbations, above which the sublinear behavior sets in $\left(N \gtrsim N_{0}\right)$. In dimensionless quantities, this is,

$$
\Phi_{0}-\Phi=A_{1} \frac{\left(\epsilon / \epsilon_{0}\right)^{3}}{1+\left(\epsilon / \epsilon_{0}\right)^{6}},
$$

where $\epsilon_{0}=0.616 \pm 0.005$ is the characteristic value above which the sublinear behavior starts to be dominant, and provides an "effective radius", $r_{0} / \epsilon \sim 3.25$, for each curvature perturbation.

In the inset of Fig. 2, we observe that by fixing the number of curvature perturbations $N=1024$ and the strength $a_{0}=2 \times 10^{-5}$, and changing the range of the perturbation, $\epsilon$ with $\lambda=8$, the difference $\Phi_{0}-\Phi$ presents a maximum for a given $\epsilon=\epsilon_{c} \sim 4$. Note that this value is similar to $\epsilon_{0}$. Due to the fact that by increasing $r_{0}$ the flux $\Phi$ decreases, one could think that the Reynolds number would achieve a plateau. However, by analysing our numerical data we have not found such behavior. Furthermore, another interesting result is that the average dimensionless curvature, here defined as $R=-10^{6} \lambda^{2}<$ $R^{\prime}>$ (where $\langle\ldots>$ means average over space), shows the same qualitative behavior. Since by increasing $\epsilon$ the metric tensor components decrease monotonically, this maximum is due to the Christoffel symbols (or non-inertial forces), which can be characterized via $R$. However, the maxima are slightly shifted, due to the fact that the Ricci scalar does not uniquely determine the metric tensor and Christoffel symbols, the quantities that play a key role in the fluid dynamic equations. Taking into account this effect, we can plot the flux deficit $\Phi_{0}-\Phi$ as a function of $R$, and find that, indeed, for $\epsilon<\epsilon_{c}$, the flux decreases by increasing the average curvature $R$ with a different law than for the case of large values of $\epsilon>\epsilon_{c}$ (see inset of Fig. 3). This gives rise to a loop-shaped curve, the reason for this behavior 
being that the metric tensor is different for $\epsilon<\epsilon_{c}$ and $\epsilon>\epsilon_{c}$, even if $R$ takes the same value. However, in both cases, the system shows that higher values of the average curvature $R$ always result in a lower flux. The behavior of the flux for $\epsilon<\epsilon_{c}$ is well represented by the following law:

$$
\Phi_{0}-\Phi=A_{2} \frac{R}{R_{0}}\left(1+\frac{R}{R_{0}}\right),
$$

and for the case of $\epsilon>\epsilon_{c}$,

$$
\Phi_{0}-\Phi=A_{3} \sqrt{\frac{R}{R_{0}+R}}+\Phi^{\prime},
$$

where $R_{0}=5.2 \pm 0.1, A_{2}=50 \pm 2, A_{3}=154 \pm 4$, and $\Phi^{\prime}=5 \pm 1$. The quantity $R_{0}$ is related to the maximum curvature achieved by the system and the intersection of the two laws (see Fig. 3). The other interesting quantity is $\Phi^{\prime}$, which represents the difference of flux between $\epsilon \gg \epsilon_{c}$ and $\epsilon \ll \epsilon_{c}$, when the curvature scalar becomes zero, and it is due to the fact that in both cases, although the space has no curvature, it has nonetheless different metric tensors.

Finally, in order to give a more general description to our campylotic medium, we perform a detailed study of the the flux $\Phi$, as a function of two dimensionless number, $R e$ and $\epsilon$. Since $R e$ is not the only dimensionless number that characterizes the flow, we can expect different behaviors depending on how the $R e$ is varied. Therefore, we will change $R e$ by modifying both, the perturbation range $r_{0}$ and the viscosity $\mu$, separately. In Fig. 4, we can appreciate the dependence of the flux deficit, $\Phi_{0}-\Phi$, as a function of $\epsilon$ for a fix Reynolds number, $R e=3$. Note that fixing the Reynolds number and changing the ratio $\epsilon$, implies to vary the number of impurities perturbations. As a consequence, in order to achieve a broad range of values, we decrease the amplitude $a_{0}$, taking the value of $2 \times 10^{-6}$. In the domain $\epsilon \in[1,10]$, we found that the flux decreases following a power law with exponent 3 .

In the inset of Fig. 4, we report the behavior of the flux deficit as a function of the Reynolds number by changing $r_{0}$, for $\epsilon=5$ and different values of the fluid viscosity $\mu$. Here, in order to vary $R e$, via $r_{0}$, for fixed $\epsilon$ and $\mu$, we need to modify both, $N$ and $r_{0}$. In the regime of Reynolds numbers studied in this work, and for a fixed viscosity, the flux increases with the Reynolds number almost linearly upto some critical number, $R e_{c}$, above which a sublinear behavior is found

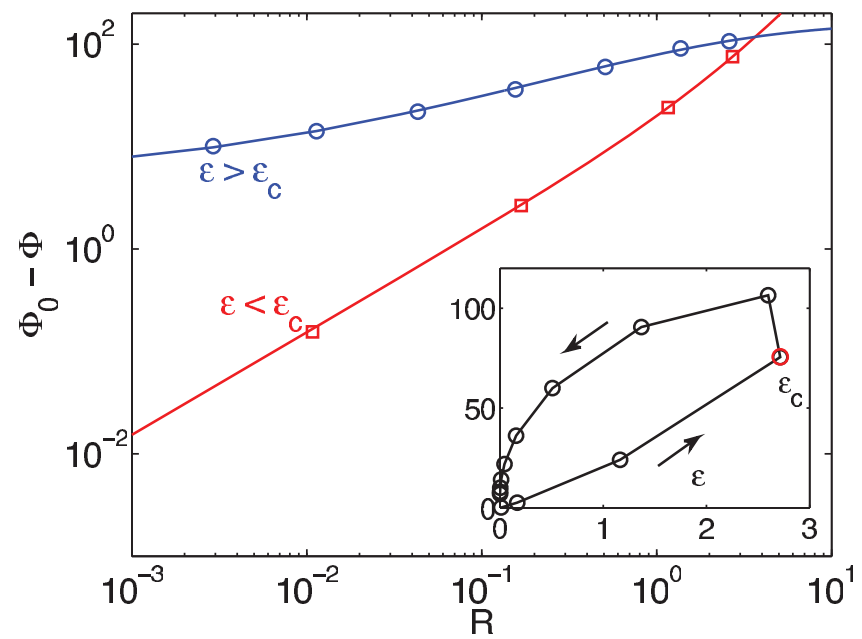

Figure $3 \mid$ Flux deficit, $\Phi_{0}-\Phi$, as a function of the average curvature, $R$, for large and small values of $\epsilon$. We have fixed $a_{0}=0.00002, N=1024(R e$ $\sim 1 \ldots$ 1000). The solid lines denote the analytical curves according to Eqs. (8) and (9). The inset shows the loop which arises by parametrizing the flux-curvature relation in terms of $\epsilon$. Here, $\epsilon_{c}$ is the radius at which the Ricci curvature attains its maximum upon increasing $\epsilon$. The lower and upper branches correspond to $\epsilon<\epsilon_{c}$ and $\epsilon>\epsilon_{c}$, respectively. nearing a plateau. The flux deficit obeys the law,

$$
\frac{\Phi_{0}-\Phi}{\Phi_{0}}=\frac{B_{2}}{1+\left(\operatorname{Re} / \operatorname{Re}_{c}\right)^{3}},
$$

with $B_{2}=0.259 \pm 0.006$ and $R e_{c}=5.8 \pm 0.1$ for $\mu=0.18, B_{2}=0.289$ \pm 0.008 and $R e_{c}=0.75 \pm 0.02$ for $\mu=0.55$, and $B_{2}=0.16 \pm 0.005$ and $R e_{c}=35.8 \pm 0.9$ for $\mu=0.07$. Note that Eq. (10) is satisfied regardless the viscosity of the fluid, and we can conclude that by decreasing the viscosity, we increase the critical Reynolds number at which the sublinear regime begins. On the other hand, the insensitivity of the flux deficit at large Reynolds numbers (by changing the interaction range, $r_{0}$, and therefore, large values of $r_{0}$ ) is due to the fact that the average curvature of the system decreases, leading to a flat space, and $\Phi \rightarrow\left(1+B_{2}\right) \Phi_{0}$.

\section{Discussion}

Summarizing, we have explored the laws that rule the flow through campylotic media consisting of randomly distributed curvature perturbations, and shown that, for the configurations studied in this work, curved spaces invariably support less flux than flat spaces. Furthermore, the flux can be characterized by the Ricci scalar, a geometrical invariant that contains the metric tensor and Christoffel symbols. The trajectories of the flow can become very complicated, due to the total curvature of the medium, presenting, in some cases, orbits winding several times around regions with high curvature. To add generality to our study, we have also analyzed the flux transport across the campylotic medium as a function of dimensionless numbers, $\epsilon$ and $R e$. Furthermore, we have found that the different laws that characterize the campylotic medium are valid regardless of the viscosity of the fluid, as far as the laminar regime holds. Indeed, further extensions to consider turbulent flows will be a subject of future research.

To calculate the flux in campylotic media, we have developed a new lattice Boltzmann model to simulate fluid dynamics in curved manifolds using an arbitrary coordinate system. The model has been successfully validated (see SI) on the Taylor-Couette instability for the case of two concentric cylinders and spheres, the inner rotating with a given speed and the outer being fixed. We also studied the Taylor-Couette instability in two concentric rotating tori, finding

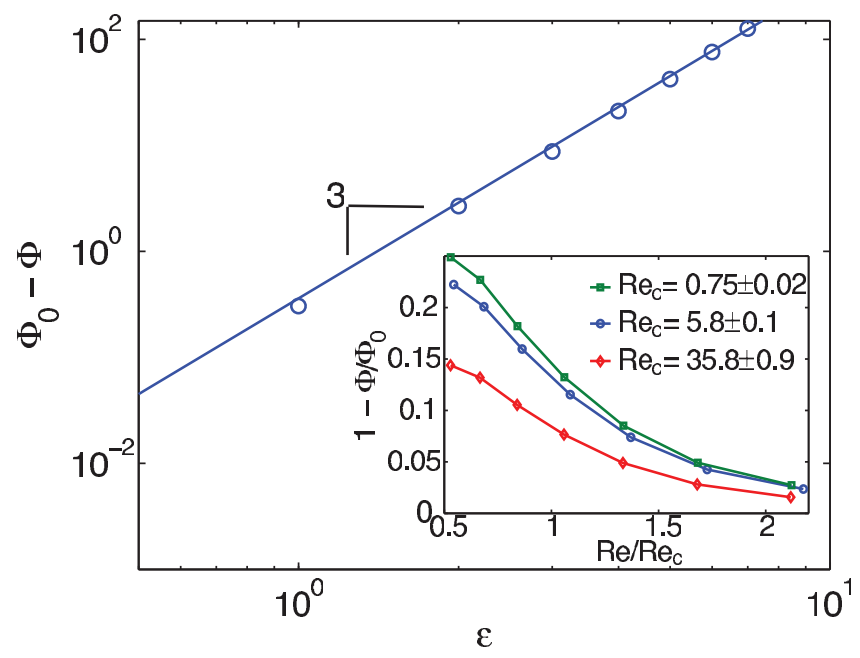

Figure $4 \mid$ Flux as a function of the dimensionless numbers Re and $\epsilon$. Flux deficit, $\Phi_{0}-\Phi$, as a function of the dimensionless number $\epsilon$ for $R e=3$, which decreases following a power law with exponent 3 , in the studied regime. In the inset, we can observe the dependence on $R e$ by fixing $\epsilon=5$ and varying $r_{0}$, for different viscosities, $v=0.55$ (green squares), 0.18 (blue circles), and 0.07 (red diamonds). 
that the critical Reynolds number for the onset of the instability is about ten percent larger than the one for the cylinder. By solving the Navier-Stokes equations for curved spaces in contravariant coordinates, which can be represented on a cubic lattice precisely in the format requested by the lattice Boltzmann formulation, the present model opens up the possibility to study fluid dynamics in complex manifolds by retaining the outstanding simplicity and computational efficiency of the standard lattice Boltzmann method in cartesian coordinates.

\section{Methods}

In order to formulate a corresponding lattice Boltzmann model, we implement an expansion of the Maxwell-Boltzmann distribution, Eq. (1), in Hermite polynomials, so as to recover the moments of the distribution function up to third order in velocities, as it is needed to correctly reproduce the dissipation term in the hydrodynamic equations. The expansion of the Maxwell-Boltzmann distribution was introduced by Grad in his 13 moment system ${ }^{22}$. Since this expansion is performed in velocity space, and the metric only depends on the spatial coordinates, we expect such an expansion to preserve its validity also in the case of a general manifold. We have followed a similar procedure as the one described in Refs. 23, 24.

For the discretization of the Maxwell Boltzmann distribution (1) and the Boltzmann equation (5), we need a discrete velocity configuration supporting the expansion up to third order in Hermite polynomials. Our scheme is based on the D3Q41 (three dimensions and 41 velocity vectors) lattice proposed in Ref. 25, which corresponds to the minimum configuration supporting third-order isotropy in three spatial dimensions, along with a $\mathrm{H}$-theorem for future entropic extension $\mathrm{s}^{26}$ of the present work.

In the following, we shall use the notation $c_{\lambda}^{i}$ to denote the $i$-th contravariant component of the vector numbered $\lambda$. Thus, the discrete Boltzmann equation for our model takes the form, $f_{\lambda}\left(x^{i}+c_{\lambda}^{i} \delta t, t+\delta t\right)-f_{\lambda}\left(x^{i}, t\right)=-\frac{\delta t}{\tau}\left(f_{\lambda}-f_{\lambda}^{\mathrm{eq}}\right)+\delta t \mathcal{F}_{\lambda}$, where $\mathcal{F}_{\lambda}$ is the forcing term, which contains the Christoffel symbols, and $f_{\lambda}^{\text {eq }}$ is the discrete form of the Maxwell-Boltzmann distribution, Eq. (1). The relevant physical information about the fluid and the geometry of the system is contained in these two terms. The macroscopic variables are obtained according to the relations, $\rho=\sum_{\lambda=0}^{41} f_{\lambda}, \rho u^{i}=\sum_{\lambda=0}^{41} f_{\lambda} c_{\lambda}^{i}$.

The cell configuration $D 3 Q 41$ has the discrete velocity vectors: $(0,0,0),( \pm 1,0,0)$, $( \pm 1, \pm 1,0),( \pm 1, \pm 1, \pm 1),( \pm 3,0,0),(0, \pm 3,0),(0,0, \pm 3)$, and $( \pm 3, \pm 3, \pm 3)$. The speed of sound for this configuration is $c_{s}^{2}=1-\sqrt{2 / 5}$. With this setup, and taking into account that the vectors $\xi^{i}$ and $u^{i}$ are normalized by the speed of sound, we obtain the following discrete equilibrium distribution,

$$
\begin{aligned}
f_{\lambda}^{\mathrm{eq}} & =w_{\lambda} \rho\left(\frac{5}{2}+2 \frac{c_{\lambda}^{i} u^{i}}{c_{s}^{2}}+\frac{1}{2} \frac{c_{\lambda}^{i} g^{i j} c_{\lambda}^{j}}{c_{s}^{2}}-\frac{1}{2} \frac{c_{\lambda}^{i} c_{\lambda}^{i}}{c_{s}^{2}}+\frac{1}{2} \frac{\left(c_{\lambda}^{i} u^{i}\right)^{2}}{c_{s}^{4}}\right. \\
& -\frac{g^{i i}}{2}-\frac{u^{i} u^{i}}{2 c_{s}^{2}}+\frac{\left(c_{\lambda}^{i} u^{i}\right)^{3}}{6 c_{s}^{6}}+\frac{1}{2} \frac{\left(c_{\lambda}^{i} u^{i}\right)}{c_{s}^{4}}\left(c_{\lambda}^{j} g^{j k} c_{\lambda}^{k}-c_{\lambda}^{j} c_{\lambda}^{j}\right) \\
& \left.-\frac{\left(c_{\lambda}^{i} u^{i}\right)\left(u^{j} u^{j}\right)}{2 c_{s}^{4}}-\frac{\left(c_{\lambda}^{i} u^{i}\right)}{2 c_{s}^{2}}\left(g^{j j}-3\right)-\frac{u^{i} g^{i j} c_{\lambda}^{j}}{c_{s}^{2}}\right)
\end{aligned}
$$

where the weights $w_{\lambda}$ are defined as, $w_{(0,0,0)}=\frac{2}{2025}(5045-1507 \sqrt{10})$,

$w_{(1,0,0)}=\frac{37}{5 \sqrt{10}}-\frac{91}{40}, w_{(1,1,0)}=\frac{1}{50}(55-17 \sqrt{10}), w_{(1,1,1)}=\frac{1}{1600}(233 \sqrt{10}-730)$, $w_{(3,0,0)}=\frac{1}{16200}(295-92 \sqrt{10})$, and $w_{(3,3,3)}=\frac{1}{129600}(130-41 \sqrt{10})$. The forcing term takes the form

$$
\begin{aligned}
\delta t \mathcal{F}_{\lambda} & =w_{\lambda} \rho\left[( g ^ { k l } - \delta ^ { k l } + \frac { u ^ { k } u ^ { l } } { c _ { s } ^ { 2 } } ) \left(\frac{c_{\lambda}^{k} c_{\lambda}^{l} c_{\lambda}^{i} F_{\lambda}^{i}}{2 c_{s}^{4}}-\frac{c_{\lambda}^{m} F_{\lambda}^{m} \delta^{k l}}{2 c_{s}^{2}}\right.\right. \\
& \left.\left.-\frac{c_{\lambda}^{l} F_{\lambda}^{k}}{2 c_{s}^{2}}-\frac{c_{\lambda}^{k} F_{\lambda}^{l}}{2 c_{s}^{2}}\right)+\frac{c_{\lambda}^{i} F_{\lambda}^{i}}{c_{s}^{2}}+\frac{\left(c_{\lambda}^{l} u^{l}\right) c_{\lambda}^{i} F_{\lambda}^{i}}{c_{s}^{4}}-\frac{u^{i} F_{\lambda}^{i}}{c_{s}^{2}}\right],
\end{aligned}
$$

with $F_{\lambda}^{i}=-\Gamma_{j k}^{i} \xi_{\lambda}^{j} \xi_{\lambda}^{k}$ and $\delta^{k l}$ the Kronecker delta. In the presence of an external force $F_{\text {ext }}$, this simply extends to $F_{\lambda}^{i} \rightarrow F_{\lambda}^{i}+F_{\text {ext. }}^{i}$.

In order to recover the correct macroscopic fluid equations, via a ChapmanEnskog expansion, the other moments, Eqs. (2), (3), and (4), also need to be reproduced. A straightforward calculation shows that the equilibrium distribution function $f_{\lambda}^{\text {eq }}$ meets the requirement. The shear viscosity of the fluid can also be calculated as $\mu=\rho(\tau-1 / 2) c_{s}^{2} \delta t$. In this way one can calculate the fluid motion in spaces with arbitrary local curvatures.

We have measured the efficiency vs. standard LB and the resulting overhead (about 3 ) is almost entirely to be ascribed to the fact that, by relativistic necessity (third order moments to be matched), we work with 41 velocities. Although a detailed head-on comparison remains to be done in future work, such overhead appears perfectly acceptable, especially in view of future applications to relativistic dissipative hydrodynamics in highly complex manifolds. More details about the discretization of this model on a lattice and the numerical validation can be found in the SI.

All simulations in this paper are performed using the smallest system size that keeps the physical results unchanged at increasing the grid resolution. The reason for this choice is that we need to calculate the metric tensor and the Christoffel symbols locally, which calls for a computational compromise. On the one hand, storing the metric tensor and Christoffel symbols as arrays, minimizes the CPU time, at the price of increasing memory requirements. On the other hand, by computing these quantities "on the fly", memory request is significantly reduced, to the detriment of computational time. For the simulations presented in this work, we have used the former approach (metric and Christoffel symbols as stored as arrays), so that we cannot compute very large system sizes in a reasonable computational time. However, finding the optimal tradeoff between the two approaches above will be a subject of future work.

1. Landau, L. D. \& Lifshitz, E. M. The classical theory of fields, by Landau, L. D. \& Lifshitz, E. M. (Pergamon Press; Addison-Wesley Pub. Co., Oxford, Reading, Mass., 1962), rev. 2d ed. edn.

2. Seychelles, F., Amarouchene, Y., Bessafi, M. \& Kellay, H. Thermal convection and emergence of isolated vortices in soap bubbles. Phys. Rev. Lett. 100, 144501 (2008)

3. Priest, E. Solar magneto-hydrodynamics. Geophysics and astrophysics monographs (D. Reidel Pub. Co., 1984).

4. Mullin, T. \& Blohm, C. Bifurcation phenomena in a taylor-couette flow with asymmetric boundary conditions. Phys. of Fluids 13, 136-140 (2001).

5. Di Prima, R. \& Swinney, H. Instabilities and transition in flow between concentric rotating cylinders, vol. 45 of Topics in Applied Physics (Springer Berlin/ Heidelberg, 1985).

6. Bartels, F. Taylor vortices between two concentric rotating spheres. J. Fluid Mech. 119, 1-25 (1982)

7. Johnson, G., Borovetz, H. \& Anderson, J. A model of pulsatile flow in a uniform deformable vessel. J. Biomech. 25, 91-100 (1992).

8. Ottaviani, M. et al. Numerical simulations of ion temperature gradient-driven turbulence. Phys. Fluids B 2, 67 (1990) http://dx.doi.org/10.1063/1.859540.

9. Bear, J. Dynamics of Fluids in Porous Media (American Elsevier Publising Company, 1972).

10. Schrauf, G. The first instability in spherical taylor-couette flow. Journal of Fluid Mechanics 166, 287-303 (1986).

11. Hirsch, C. Numerical Computation of Internal and External Flows: Fundamentals of Computational Fluid Dynamics. No. Bd. 1 in Butterworth Heinemann (Butterworth-Heinemann, 2007).

12. Chen, H. et al. Extended Boltzmann kinetic equation for turbulent flows. Science 301, 633-636 (2003).

13. Marcus, P. \& Tuckerman, L. Simulation of flow between concentric rotating spheres. part 1. steady states. J. Fluid Mech. 185 (1987).

14. Baumgarte, T. W. \& Shapiro, S. L. Numerical integration of einstein's field equations. Phys. Rev. D 59, 024007 (1998)

15. Schnetter, E., Hawley, S. H. \& Hawke, I. Evolutions in 3 d numerical relativity using fixed mesh refinement. Classical and Quantum Gravity 21, 1465 (2004).

16. Mendoza, M., Boghosian, B. M., Herrmann, H. J. \& Succi, S. Fast lattice Boltzmann solver for relativistic hydrodynamics. Phys. Rev. Lett. 105, 014502 (2010).

17. Mendoza, M., Herrmann, H. J. \& Succi, S. Preturbulent regimes in graphene flow. Phys. Rev. Lett. 106, 156601 (2011).

18. Love, P. J. \& Cianci, D. From the Boltzmann equation to fluid mechanics on a manifold. Philosophical Transactions of the Royal Society A: Mathematical, Physical and Engineering Sciences 369, 2362-2370 (2011).

19. Sinitsyn, A., Dulov, E. \& Vedenyapin, V. Kinetic Boltzmann, Vlasov and Related Equations (Elsevier, 2011).

20. Bini, D., Jantzen, R. T. \& Stella, L. The general relativistic poyntingrobertson effect. Classical and Quantum Gravity 26, 055009 (2009).

21. Bini, D., Gregoris, D. \& Succi, S. Radiation pressure vs. friction effects in the description of the poynting-robertson scattering process. EPL (Europhysics Letters) 97, 40007 (2012).

22. Grad, $\mathrm{H}$. Note on $\mathrm{n}$-dimensional hermite polynomials. Communications on Pure and Applied Mathematics 2, 325-330 (1949).

23. Martys, N. S., Shan, X. \& Chen, H. Evaluation of the external force term in the discrete Boltzmann equation. Phys. Rev. E 58, 6855-6857 (1998).

24. Shan, X. \& He, X. Discretization of the velocity space in the solution of the Boltzmann equation. Phys. Rev. Lett. 80, 65-68 (1998).

25. Chikatamarla, S. S. \& Karlin, I. V. Lattices for the lattice Boltzmann method. Phys. Rev. E 79, 046701 (2009).

26. Karlin, I. V., Gorban, A. N., Succi, S. \& Boffi, V. Maximum entropy principle for lattice kinetic equations. Phys. Rev. Lett. 81, 6-9 (1998).

\section{Acknowledgments}

The authors are grateful for the financial support of the Eidgenössische Technische Hochschule Zürich (ETHZ) under Grant No. 06 11-1, and the European Research Council (ERC) Advanced Grant 319968-FlowCCS. 


\section{Author contributions}

M.M., S.S. and H.J.H. conceived and designed the research, analyzed the data, worked out the theory, and wrote the manuscript. All authors reviewed the manuscript.

\section{Additional information}

Supplementary information accompanies this paper at http://www.nature.com/ scientificreports
Competing financial interests: The authors declare no competing financial interests.

How to cite this article: Mendoza, M., Succi, S. \& Herrmann, H.J. Flow Through Randomly Curved Manifolds. Sci. Rep. 3, 3106; DOI:10.1038/srep03106 (2013).

(c) (i) $\odot$ This work is licensed under a Creative Commons Attribution(c) ${ }_{\mathrm{BY}} \mathrm{NC}$ ND NonCommercial-NoDerivs 3.0 Unported license. To view a copy of this license, visit http://creativecommons.org/licenses/by-nc-nd/3.0 